\title{
Processo de Enfermagem como potencializador da prática da acupuntura $^{a}$
}

\author{
Nursing Process as a potentializer of acupuncture practice \\ Proceso de Enfermería como potencializador de la práctica de acupuntura
}

Ana Paula Senna Bousfield ${ }^{1}$ (C) Maria Itayra Padilha ${ }^{2}$ (1) Maria Lígia dos Reis Bellaguarda ${ }^{1}$ (B) Roberta $\operatorname{Costa}^{1}$ (1)

1. Universidade Federal de Santa Catarina. Florianópolis, SC, Brasil.

2. Escola Paulista de Enfermagem. São Paulo, SP, Brasil.
Autor correspondente:

Ana Paula Senna Bousfield.

E-mail: paula.bousfield@gmail.com

Recebido em 13/05/2020.

Aprovado em 11/02/2021.

DOI:https://doi.org/10.1590/2177-9465-EAN-2020-0148

\section{Resumo}

Objetivo: Analisar a utilização do Processo de Enfermagem na prática da acupuntura, no período de 1997 a 2015. Método: Qualitativo com abordagem Histórico-Social, por meio da história oral temática aplicada a 20 enfermeiras especialistas em acupuntura fundamentado na Sociologia das Profissões, de Eliot Freidson. Da análise temática, construíram-se duas categorias: Processo de Enfermagem como organizador da prática da acupuntura; e Desconhecimento da importância do Processo de Enfermagem na prática da acupuntura. Resultados: A aplicação do Processo de Enfermagem na consulta de enfermagem, com foco na prática da acupuntura como cuidado e tratamento, possibilita uma atuação diferenciada, maior compreensão e reconhecimento diagnóstico, escolha mais adequada de técnicas favoráveis à qualidade e o bem-estar dos usuários, além de consolidar a sua utilização como prática especializada. Considerações finais: O Processo de Enfermagem possibilita um avanço tecnológico aplicado na consulta de enfermagem, ao se implementar a acupuntura como tratamento e reabilitação, colaborando na evolução e prognóstico no atendimento, e uma estratégia facilitadora para a enfermeira acupunturista junto ao cliente.

Palavras-chave: Acupuntura; Enfermagem; Processo de Enfermagem; História da Enfermagem; Especialização.

\section{Abstract}

Objective: To analyze the use of the Nursing Process in the practice of acupuncture, from 1997 to 2015 . Method: A qualitative, with a Social-Historical approach, through thematic oral history applied to 20 nurses specialized in acupuncture, based on Eliot Freidson's Sociology of Professions. From the thematic analysis, two categories were constructed: Nursing Process as an organizer of acupuncture practice; and Unawareness of the importance of the Nursing Process in acupuncture practice. Results: The application of the Nursing Process in the nursing consultation, focusing on the practice of acupuncture as care and treatment, enables a differentiated performance, greater understanding and diagnostic recognition, more appropriate choice of techniques favorable to the quality and well-being of users, and consolidates its use as a specialized practice. Final Considerations: The Nursing Process enables a technological advance applied in the nursing consultation, when implementing acupuncture as treatment and rehabilitation, collaborating in the evolution and prognosis in the care, and a facilitating strategy for the acupuncturist nurse with the client.

Keywords: Acupuncture; Nursing; Nursing process; History of Nursing; Specialization.

\section{Resumen}

Objetivo: Analizar el uso del Proceso de Enfermería en la práctica de la acupuntura de 1997 a 2015. Método: Cualitativo con abordaje Histórico-Social, a través de la historia oral temática aplicada a 20 enfermeras especializadas en Acupuntura, basado en la sociología de las profesiones de Eliot. Freidson. A partir del análisis temático se construyeron dos categorías: Proceso de Enfermería como organizador de la práctica de la acupuntura; y desconocimiento de la importancia del Proceso de Enfermería en la práctica de la acupuntura. Resultados: La aplicación del Proceso de Enfermería en la consulta de enfermería con un enfoque en la práctica de la acupuntura como cuidado y tratamiento, posibilita un desempeño diferenciado, mayor comprensión y reconocimiento diagnóstico, elección más adecuada de técnicas que favorezcan la calidad y el bienestar de los usuarios, además de consolidar su uso como práctica especializada. Consideraciones finales: El proceso de Enfermería permite un avance tecnológico aplicado en la consulta de enfermería con la implementación de la acupuntura como tratamiento y rehabilitación, colaborando en la evolución y pronóstico en el cuidado y una estrategia facilitadora para la Enfermera Acupunturista con el cliente.

Palabras clave: Acupuntura; Enfermería; Proceso de Enfermería; Historia de la Enfermería; Especialización. 


\section{INTRODUÇÃO}

A análise da condição de saúde e bem-estar do indivíduo é uma das responsabilidades da enfermeira, sendo que, para operacionalizar essa análise, ela se utiliza do Processo de Enfermagem (PE). Na prática assistencial, gerencial, de ensino e pesquisa em enfermagem e saúde, o PE emergiu na década de 1950, nos Estados Unidos, para qualificar o cuidado de enfermagem. ${ }^{1}$ Trata-se de uma metodologia de trabalho inserida na prática clínica, e ensinada na formação da enfermeira, na década de 1970, sendo, até a atualidade, uma potência à evolução da sua prática profissional. ${ }^{2}$

O Processo de Enfermagem é uma estratégia de organização e sistematização do cuidado prestado pela enfermagem. $\mathrm{Na}$ literatura, ainda se pode encontrar outras denominações, como Sistematização da Assistência de Enfermagem (SAE) e Metodologia da Assistência de Enfermagem (MAE), termos que já foram utilizados, porém não se constituem sinônimos. ${ }^{3}$

A Sistematização da Assistência em Enfermagem é ferramenta que auxilia na definição do papel do enfermeiro, propiciandoIhe usar seus saberes técnico-científicos e humanitários na assistência ao indivíduo, e comprovar seu exercício profissional com a operacionalização do Processo de Enfermagem. ${ }^{4}$

O PE foi idealizado e implementado no Brasil pela Dra. Wanda de Aguiar Horta, teórica brasileira, a qual criou uma base metodológica para essa prática. Os escritos de Horta propiciaram o desenvolvimento da enfermagem e a disseminação das Teorias de Enfermagem, baseadas nos modelos americanos. Vale ressaltar que tais teorias distinguem, indicam e confrontam conceitos expressivos de fenômenos que são de interesse exclusivo da profissão do enfermeiro. Em conformidade com outros países, sua implantação iniciou nas Escolas de Enfermagem, como parte dos currículos de graduação. ${ }^{4}$

O registro da assistência de enfermagem sob sistemática de análise reflete no processo de trabalho, baseado em uma visão ampliada de cuidado aos pacientes e famílias, em continuidade, com amparo legal e facilitador na comunicação multidisciplinar. ${ }^{5}$

O Processo de Enfermagem foi reconhecido como instrumento de trabalho e atribuição do enfermeiro a partir da aprovação da Lei do Exercício Profissional, de 1986 (Lei n.ำ 7.498/86 e Decreto n. 94.406/87), mediante a Resolução COFEN n.ำ 272/2002, que determinou a obrigatoriedade da Sistematização da Assistência de Enfermagem em todo o território nacional. Posteriormente, foi substituída pela Resolução $n$. o 358/2009, a qual dispõe que o Processo de Enfermagem deve estar sustentado em um suporte teórico que auxilie a coleta de dados, a realização de diagnósticos e o planejamento das ações de enfermagem, além de fornecer a base para a avaliação dos resultados. ${ }^{6}$

A inserção no processo de trabalho das enfermeiras nas instituições e serviços de saúde, no Brasil, tem sido paulatina e enfrenta algumas dificuldades, apontadas na literatura da área. As fragilidades são recorrentes na consolidação do processo relacionado à dinâmica assistencial da enfermagem, no tocante a recursos humanos e preparo do pessoal e da equipe, sobrecarga de trabalho, práticas ainda focadas na tarefa, entre outras dificuldades na condição laboral. ${ }^{5-7}$

A enfermagem deve incluir as práticas integrativas e complementares como um protótipo de cuidado a ser doutrinado e aplicado no respectivo ambiente, considerando as intervenções biomédicas e farmacológicas que, em sua maioria, apresentam muitos efeitos colaterais e são excessivamente agressivas ao organismo.

O Processo de Enfermagem é um método ativo, flexível, organizado e utilizado na prática clínica da enfermagem, com o intuito de conduzir o trabalho da enfermeira na averiguação dos dados do paciente, apontando as necessidades de cuidados, acionando intervenções e avaliando os resultados. ${ }^{8}$ Ele viabiliza, por meio de suas etapas - Histórico de Enfermagem, Diagnóstico de Enfermagem, Intervenção, Planejamento ou Implementação e Avaliação ou Evolução de Enfermagem -, compreender o paciente como um todo e desenvolver uma assistência de enfermagem humanizada, direcionada às necessidades individualizadas. Além disso, a coleta de dados ampara o Processo de Enfermagem, concedendo ao enfermeiro aplicar os conhecimentos técnico-

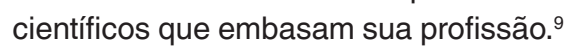

O Processo de Enfermagem é parte da Sistematização da Assistência de Enfermagem, a qual é entendida como método do trabalho da profissão, quanto à organização, pessoal e instrumentos, o que viabiliza a sua realização. A SAE antevê a definição da natureza do trabalho a ser realizado e a descrição do PE, desde a base teórico-filosófica, até o tipo de profissional, os métodos, os objetivos e os recursos materiais, para a execução do cuidado. ${ }^{4}$

A implementação do Processo de Enfermagem tem sido utilizada prioritariamente para ações específicas de enfermagem, junto ao cuidado aos usuários dos serviços de saúde hospitalares e de atenção básica. Porém, como a enfermagem é composta de várias especialidades, estas podem realizar a sua implementação, visando qualificar o cuidado prestado a esses usuários. Nesse sentido, uma enfermeira especialista em acupuntura poderia agregar seus conhecimentos acerca do Processo de Enfermagem, com os conhecimentos específicos da Acupuntura e, assim, qualificar esse tipo de atendimento potencializando o olhar para o ser humano em sua totalidade.

A acupuntura, bem como a enfermagem, fundamenta-se na proposta de integralização do cuidado à saúde, por intermédio de respostas humanas obtidas na relação do ser com o meio. Essas respostas são avaliadas mediante identificação de sinais e sintomas, com o intuito de estabelecer um diagnóstico, por associações, que, no caso da acupuntura, são utilizados os diagnósticos energéticos, e, na enfermagem, os diagnósticos de enfermagem.$^{10} \mathrm{O}$ procedimento clínico da acupuntura é semelhante ao do Processo de Enfermagem e dentro da enfermagem, e uma das áreas que proporciona à enfermeira maior autonomia enquanto profissional liberal.

A acupuntura é uma técnica de inserção e manipulação de agulhas apropriadas em pontos específicos, para fins terapêuticos, nos quais se localizam os meridianos, que são canais por onde 
passa a força vital, denominada Q̀̀. Cada meridiano está vinculado diretamente a um sistema fisiológico ou mental de cada ser. ${ }^{11} \mathrm{E}$ praticada na China há mais de 4 mil anos e é parte integrante da Medicina Tradicional Chinesa (MTC). No Brasil, a prática da MTC teve início com a vinda dos primeiros imigrantes chineses para o Rio de Janeiro, em 1810. A prática da Acupuntura foi introduzida na tabela do Sistema de Informação Ambulatorial - SIA/SUS em

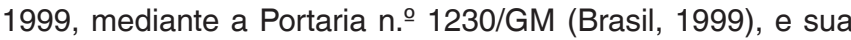
prática reforçada pela Portaria n. -971 , publicada pelo Ministério da Saúde em 2006, que aprovou a Política Nacional de Práticas Integrativas e Complementares no Sistema Único de Saúde. ${ }^{12}$

A utilização do Processo de Enfermagem possibilita a especificidade de cuidados individualizados, assim como norteia o processo decisório da enfermeira nas situações de gerenciamento da equipe. Oportuniza, também, avanços na qualidade do cuidado, o que impulsiona sua adoção nas instituições que prestam assistência à saúde. A inter-relação entre as etapas do Processo de Enfermagem nos permite planejar e replanejar à medida que mudam as necessidades dos pacientes.

A acupuntura no cuidado de enfermagem é objeto de interesse e discussão entre experts e especialistas. O número de profissionais atuantes e de produções científicas ainda é pequeno quando comparado às reais potencialidades do exercício profissional. Precisa-se construir um corpo de conhecimento específico entre a enfermagem e a acupuntura, para assim reforçar a utilização e execução do Processo de Enfermagem, ampliando seu campo de atuação de forma autônoma, segura e sólida. ${ }^{10}$

O presente estudo tem como alicerce a Sociologia das Profissões, de Eliot Freidson, sob a argumentação de três fatores que caracterizam uma profissão: autonomia, expertise e credencialismo sobre seu próprio trabalho, em que descrevem o tipo ideal de profissionalização. ${ }^{13}$

Este estudo tem por objetivo analisar a utilização do Processo de Enfermagem na prática da acupuntura, no período de 1997 a 2015. O recorte histórico é de 1997 a 2015, justificando seu início pelo reconhecimento do exercício da acupuntura para a profissional enfermeira, respaldado na Resolução COFEN no 197 , de 1997. O recorte final concentra-se em 2015, com a ampliação da Política Nacional de Práticas Integrativas e Complementares. ${ }^{14}$

O objeto de pesquisa deste estudo refere-se à inserção do Processo de Enfermagem enquanto metodologia de análise para a prática da enfermeira acupunturista. Justifica-se por abordar uma temática essencial, que é o Processo de Enfermagem como potencializador na prática da acupuntura por enfermeiras. Sendo um estudo histórico-social, permite compreender os desafios do passado e as perspectivas em relação ao futuro, para a implementação da acupuntura como parte do trabalho da enfermeira, complementando, assim, a História da Enfermagem Brasileira.

\section{MÉTODO}

Estudo qualitativo, de abordagem histórico-social, que se define como um enfoque sistemático por meio de coleta, organização e avaliação crítica de dados, relacionadas a ocorrências passadas, compreendendo no espaço temporal, os diferentes aspectos do cotidiano de classes e grupos sociais. ${ }^{15}$ A estratégia metodológica utilizada foi da História Oral Temática, como fonte primária, tratando do objeto específico vivido pelo indivíduo. ${ }^{16}$

Participaram desta pesquisa 19 enfermeiras e 1 enfermeiro com especialização em acupuntura. Para a escolha dos entrevistados, foi realizado contato com o Conselho Regional de Enfermagem de Santa Catarina (COREN/SC), a fim de conhecer e identificar as profissionais Enfermeiras Especialistas em Acupuntura. Além disso, buscaram-se enfermeiras que tivessem realizado sua formação em Acupuntura em Escolas específicas de Santa Catarina. Para maior abrangência de participantes, foi utilizado o método snowball - estratégia quando um profissional indica outro e o próximo também indica -, com o intuito de alcançar o máximo de profissionais para colaborarem na pesquisa. Como critério de inclusão, utilizamos profissionais que adquiriram o título de especialista entre 1997 e 2015, com atuação na área no período do recorte histórico; e como critério de exclusão, aqueles profissionais especialistas que atuaram por menos de três anos realizando atividades com acupuntura.

$O$ roteiro de entrevista foi composto de 16 perguntas semiestruturadas. Foram realizadas 10 entrevistas via áudio de WhatsApp e 10 presenciais, em locais determinados pelos participantes, no período de março a maio de 2018, com uma média de duração de 60 minutos cada. Todas elas foram gravadas utilizando um gravador digital, transcritas, e validadas por meio do Termo de Cessão de Entrevista. Os relatos foram analisados, comparados e agrupados por similaridade de conteúdo, visando organizar a discussão.

O método de análise temática de conteúdo proposto por Minayo ${ }^{17}$ - hermenêutico (compreensão) e dialético (crítica) foi aplicado para embasar este estudo, utilizando informações dos entrevistados para melhor compreensão desse contexto, juntamente com o referencial teórico do estudo, o qual se baseou nos preceitos da Sociologia das Profissões, propostos por Eliot Freidson. Da análise dos dados, foi originada a categoria Processo de Enfermagem como organizador da prática da acupuntura.

Este estudo foi aprovado pelo Comitê de Ética em Pesquisa com Seres Humanos, da UFSC, sob Parecer $n . \stackrel{\circ}{2} .562 .782$, do CAAE 2.562.782. Após o primeiro contato por e-mail, foram aplicados os critérios de inclusão e exclusão dos profissionais e enviados os convites para participação na pesquisa, juntamente com o Termo de Consentimento Livre e Esclarecido. Em respeito às questões éticas da autonomia e individualidade, exigida nos trabalhos com seres humanos, optou-se em identificar os participantes por nomes de pontos de acupuntura, garantindoIhes o anonimato e o sigilo de informações.

\section{RESULTADOS}

A apresentação dos resultados deste estudo mostra-se nas expressões dos participantes, em que sete utilizavam o Processo de Enfermagem na consulta de acupuntura, e 13 não o utilizavam, indicando que não era uma prática adotada como facilitador do diagnóstico e tratamento. Desses resultados, foram compostas 
duas categorias: Processo de Enfermagem como organizador da prática da acupuntura; e Desconhecimento da importância do Processo de Enfermagem na prática da acupuntura.

Na primeira categoria - Processo de Enfermagem como organizador da prática da acupuntura -, percebeu-se que, embora nem sempre as enfermeiras utilizassem todas as etapas do Processo de Enfermagem, elas demonstraram reconhecer como ele facilita a organização.

Tudo iniciou pelo Processo de Enfermagem. A visão da medicina chinesa é um pouquinho mais ampla que a nossa medicina tradicional. Eu sei que a graduação agora mudou um pouco, mas a nossa formação na época era muito voltada para um olhar integralista. Então é muito semelhante à forma como a medicina chinesa estrutura a visão de avaliação, diagnóstico do indivíduo, então eu acabo sempre associando. O diagnóstico de enfermagem está praticamente inserido, imerso nas minhas consultas. Então eu sempre digo, não faço acupuntura, eu faço a consulta de enfermagem e o atendimento clínico de acupuntura, utilizando as práticas integrativas. (Jù Què)

Tínhamos uma proposta de realizar todo registro dos atendimentos utilizando o Processo de Enfermagem. A maioria dos profissionais eram enfermeiros e os outros utilizavam outros modos de registro, mais os enfermeiros. Nós utilizávamos inclusive os diagnósticos da CIPE (Classificação Internacional para a Prática de Enfermagem); nós trabalhávamos para organizare registrar os atendimentos realizados pelos enfermeiros. (Dà Líng)

Às vezes tenho dificuldade, mas eu acho que temos bastante autonomia aqui. Existem protocolos, que em outros municípios eu nunca vi. O enfermeiro ainda tem muito que conquistar, mas, em comparação a outros municípios do estado que eu já trabalhei, estamos bem adiantados. No consultório eu não associava a SAE. (Ben Shen)

Onde eu trabalhei não era na enfermagem, era na naturologia. Lá não tem o Processo de Enfermagem, claro. Eu acho que o nosso raciocínio clínico do Processo de Enfermagem, da sistematização, nos facilita muito. Mas para o meu trabalho eu nunca usei o Processo de Enfermagem didaticamente, para atender um paciente. (Tài Xi)

O diagnóstico em acupuntura é diferenciado. Mas essa parte de captação de dados, de anamnese eu faço muito parecido, mas a parte de diagnóstico é diferente. Eu trabalho com bioenergética e cinco elementos. Hoje têm outras maneiras, mas é diferente associar um no outro. (Yún Mén)

A forma pela qual os participantes utilizaram para sistematizar o cuidado de enfermagem acupunturista seguiu uma metodologia, com um fluxo de anamnese, exame físico e clínico para alcançar os diagnósticos e, posteriormente, avaliar a condição da pessoa atendida. O Processo de Enfermagem se refere ao planejamento do cuidado de enfermagem.

A segunda categoria - Desconhecimento do Processo de Enfermagem na prática da acupuntura - apontou que muitas enfermeiras, embora até já tivessem algum tipo de informação sobre o Processo de Enfermagem, não o entendiam como parte da consulta que realizavam com os clientes atendidos, para a prática de acupuntura. Além disso, consideravam a prática da acupuntura como algo isolado da profissão de enfermagem.

Quando eu sou enfermeira, eu sou enfermeira; e quando sou acupunturista, sou acupunturista; e é bem diferente. Até certo ponto porque carrego as informações de conhecimento e acaba influenciando, ajuda muito, para fazer o diagnóstico de acupuntura. $O$ diagnóstico tem que ser com outro olhar, e não misturado assim com a medicina ocidental. Eu achava bem difícil desligar uma coisa da outra, nessa questão de Processo de Enfermagem, diagnóstico de enfermagem. Eu achava que era bem diferente a parte do conhecimento. (Ting Hui)

Quando eu paro para pensar na consulta de acupuntura e na consulta de enfermagem, eu vejo que têm semelhanças entre elas, na organização e sistematização. Mas, não que eu tenha feito isso planejado. Eu não pensei assim: eu sou enfermeira, vou fazer uma sistematização de enfermagem; mas quando eu vi, eu tinha sequência. Eu conversava e escutava paciente coletando dados, seguidamente fazia uma análise dessa situação. Escrevia a conduta, depois avaliava, mas sem utilizar a sistematização. (Shen Tang)

Não sou da época da Sistematização da Assistência. Trabalho de uma maneira diferente, claro que não consigo trabalhar sem ver a pessoa como um todo. Não adianta você tratar a unha encravada, se você não fizer uma orientação sobre o tipo de sapato que a pessoa deve usar. Tem que ver a pessoa misticamente, eu acho que isso é um uma coisa inerente à enfermagem. Cuidava para saber como ela estava inserida no meio e na sociedade em que ela vivia, como eram os relacionamentos dela. Os desequilíbrios não só acontecem por causa dos elementos internos, as outras pessoas influenciam na sua vida também. Isso eu aprendi com a enfermagem, eu aprendi 40 anos atrás desse tipo de enfermagem que sistematiza, que olha a pessoa como um todo. (Shén Mén)

Infelizmente, eu não consigo associar o processo, embora muitos atendimentos de enfermagem eu faça a conexão com a Medicina Tradicional Chinesa. Ofereço a acupuntura no meu atendimento como enfermeira, mas na clínica particular eu atendo acupuntura, e ali eu tento esquecer um pouco a minha formação de enfermeira e tento me colocar como acupunturista para não misturar. Embora seja uma mistura positiva, mas não faço. (Shen Feng) 
A ausência da integração do papel profissional como enfermeira com o papel profissional especialista em acupuntura, aponta certa dificuldade de compreensão acerca das interrelações entre o que aprendeu na formação de graduação $e$ o que aprendeu na especialização. A falta de planejamento sistematizado do atendimento de acupuntura, guiado pelo processo de enfermagem, não é percebida como parte de um mesmo corpo de conhecimentos, que não são excludentes, mas sim complementares.

\section{DISCUSSÃo}

Os fundamentos da enfermagem são os de cuidar, prevenir, promover e restabelecer; os da acupuntura são os cinco elementos, que regem o corpo humano e a natureza na qual esse corpo encontra-se inserido, resultando no cuidado, promoção e prevenção. Ou seja, a enfermagem e a acupuntura se integram em prol do bem-estar e da saúde. Essa relação organizacional na prática assistencial da acupuntura, utilizando o Processo de Enfermagem, enfatiza, diante dos resultados apresentados, o compartilhamento entre áreas do conhecimento no cuidado em saúde. Existe uma tendência para que a prática em saúde seja ampliada e ao mesmo tempo diversificada pela expertise nos múltiplos saberes e cenários.

O Processo de Enfermagem é uma ferramenta prioritária para o desenvolvimento do cuidado de enfermagem, estrutura o escopo científico da profissão, unifica a linguagem, promove a autonomia e qualifica o cuidado prestado junto aos usuários. ${ }^{18}$

Na Medicina Tradicional Chinesa, o Diagnóstico Energético é de fundamental importância para a prática da acupuntura. Em uma consulta de enfermagem, a enfermeira acupunturista pode e deve fazer uso de ambos os diagnósticos. ${ }^{19} \mathrm{O}$ Processo de Enfermagem individualiza o cuidado, reúne informações indicando diagnósticos, subsidia o planejamento de atividades para assistir as pessoas nas necessidades humanas afetadas, descreve as intervenções primordiais ao cuidado e oportuniza evoluir a condição de saúde a cada consulta. Agregar o Processo de Enfermagem ao atendimento de acupuntura pode ser considerado um dos avanços no cuidado prestado e base de organização das informações acerca dos pacientes e famílias. ${ }^{10}$

Associar o Processo de Enfermagem à prática da enfermeira acupunturista auxilia na organização do cuidado, potencializando a qualidade do atendimento e a relação profissional-paciente. Fortalece, dessa maneira, a competência e a autonomia profissional para o cuidado individualizado e específico, e reitera a importância do cuidado em saúde ser compartilhado entre profissões e especialidades.

A Sociologia das Profissões, de Eliot Freidson, fundamenta esta inter-relação de especialidades na área da saúde, quando aponta o conhecimento próprio de uma ocupação, a expertise, caracterizando-a como profissão. Assim, no âmbito da Enfermagem, em consonância com os conceitos de Freidson, para uma ocupação ser considerada profissão, necessita ter conhecimento próprio. Nesse sentido, o Processo de Enfermagem é a expertise teorizada e prática do fazer da enfermeira e, neste estudo, corrobora a prática da enfermeira acupunturista. ${ }^{20}$ Observa-se que a maioria das enfermeiras acupunturistas não utiliza 0 Processo de Enfermagem na sua prática, mas reconhece a sua importância na organização do cuidado.

Outrossim, de acordo com a Sociologia das Profissões, a autonomia é outro fator preponderante para uma profissão. No universo da acupuntura, pode-se considerar que esta garante a autonomia pela especificidade do trabalho. Para tanto, necessita da realização da consulta de enfermagem a fim de chegar a um diagnóstico, planejamento e intervenção e, assim, à existência de protocolos nacionais, que validem essa dinâmica. ${ }^{21}$ Tal inserção da prática da prescrição de enfermagem, nessa discussão, se deve a um exemplo da autonomia e compartilhamento de ações no contexto da atenção à saúde, e considera o profissionalismo da enfermeira acupunturista. Nesse sentido, no desenvolvimento do Processo de Enfermagem na acupuntura, as condutas precisam ser estabelecidas no cumprimento da SAE.

O Processo de Enfermagem é estratégia de ação e contribui para o avanço da profissionalização, de maneira que se configura como um modo de efetivar o fazer profissional relacionando conhecimento, expertise e autonomia. A execução da SAE possibilita a construção e utilização de um corpo de conhecimento próprio da enfermagem. Contribui dessa forma, para um fazer profissional autônomo, condição essencial ao reconhecimento da identidade profissional pela sociedade. ${ }^{22}$ Essa implementação aponta um diferencial para realizar o cuidado a partir da acupuntura, demonstrando a autoridade no gerenciamento da divisão de trabalho da profissão.

A identidade profissional da enfermagem ocorre por meio da construção de uma identidade política, ética e científica que expresse a responsabilidade e a autonomia desse profissional. ${ }^{23}$ $\mathrm{Na}$ literatura, apesar da lacuna significativa quanto à temática da acupuntura e o processo de enfermagem, aponta-se que a relação diagnóstica e intervenções de enfermagem aceitam prescrições envolvendo a acupuntura. ${ }^{10}$

Esta pesquisa reforça a utilização do Processo de Enfermagem na assistência por meio da acupuntura, o que pode ser desenvolvida pela enfermeira ou indicada por outro profissional. Mesmo sendo a acupuntura uma intervenção oriental tradicional chinesa, e o Processo de Enfermagem uma metodologia de trabalho e cuidado individualizado de origem ocidental, as enfermeiras acupunturistas participantes desta investigação apresentaram consenso da proximidade, da complementariedade desses conhecimentos e práticas.

De acordo com o Conselho Federal de Enfermagem, a formação latu sensu em Acupuntura, pela enfermeira, é regulamentada no Brasil. Segue a Organização Mundial da Saúde, que, a partir de avaliações de casos clínicos da acupuntura, destaca 43 agravos e doenças com possibilidade de tratamento por meio dessa intervenção. No contexto das dependências químicas, reabilitação pós-Acidente Vascular Encefálico, dores de origem lombar e cefálica são algumas condições que a acupuntura é referendada por consenso do Instituto Nacional de Saúde norte-americano. ${ }^{6}$ 
Nessa perspectiva, vê-se que a identidade da enfermeira está atrelada à qualidade e continuidade do cuidado, autônomo e fundamentado em referenciais teórico-metodológicos. A relação Medicina Tradicional Chinesa e a assistência baseada em evidências da terapêutica ocidental apresenta congruência para o registro e avaliação de saúde, uma vez que reações fisiológicas orgânicas desencadeiam ameaça à homeostase. Isso implica desequilíbrio energético e evidência paralelo na MTC em consonância com o problema de saúde apresentado. ${ }^{24}$ Essa relação dá origem a desequilíbrios internos - como emoções e reações psicológicas - e a distribuição energética mostra-se fragilizada. Tal compreensão nos leva a entender e avaliar a saúde das pessoas de forma integral.

Corroborando a isso, está o reconhecimento, por enfermeiras acupunturistas mais experientes, do Processo de Enfermagem como visão integral do paciente na ação do cuidado. A acupuntura como especialidade da enfermeira reflete um importante argumento na busca pelo conhecimento e empoderamento profissional, valores que, para Freidson, se correspondem. As profissões, para alcançarem o reconhecimento social, buscam a expertise na autonomia de seu processo de trabalho, com o intuito de deter o poder profissional sobre determinada ocupação. ${ }^{25}$

Sendo assim, a utilização do Processo de Enfermagem como organizador da prática da enfermeira acupunturista é considerado um dos avanços no cuidado, possibilitando uma prática sistematizada e de propósito de cuidado integral. Ademais, garante coerência no atendimento prestado como forma de facilitar tanto o diagnóstico quanto a organização do cuidado, a inovação e o diferencial no atendimento.

As enfermeiras são profissionais autônomas e, no âmbito dessa autonomia, há a pertinência de tal prática. A enfermeira, enquanto membro de uma equipe, não trabalha nem toma decisões sozinho. ${ }^{26}$ Por sua vez, a enfermeira apropriada da sua expertise - nesse caso, a acupuntura - apresenta autonomia e toma as decisões de forma independente e de acordo com seus conhecimentos. A autonomia profissional é uma especificidade que confere poder à profissão, atingindo o seu status quando executa o fazer sob a proteção da sociedade e do controle estatal. ${ }^{27}$

Aderir ao Processo de Enfermagem, à consulta de acupuntura, garante à enfermeira um atendimento diferenciado, pois oferece uma inovação. Diante da discussão e de acordo com Freidson, ${ }^{20}$ os três fatores que narram o tipo ideal de profissionalização autonomia, expertise e credencialismo - vão ao encontro dos avanços da enfermeira acupunturista. Além disso, tal inovação confere status à profissional, uma vez que está sustentada em autorregulação e influenciará o meio social das profissões da saúde, reiterando a valorização da enfermagem e dos membros profissionais a partir de práticas diferenciadas e qualificadas de cuidado. ${ }^{28}$

O Processo de Enfermagem é uma expertise adquirida por intermédio da experiência e conhecimento científico, o que proporciona ao profissional sua autonomia. Os diagnósticos de enfermagem podem sofrer intervenções mediante a acupuntura, sendo aplicada tanto por enfermeiros especialistas quanto indicada por outros não especialistas, mas que reconheçam nela uma possibilidade interventiva. ${ }^{29}$

O aprimoramento da prática da acupuntura e a qualificação pelo saber adquirido na formação da enfermeira expressam uma conjunção de fazeres e saberes, uma vez que a competência se estabelece pelo conhecimento, habilidades e atitudes, que resultam em mudanças na prática profissional. A enfermeira que atua com MTC está diretamente ligada às ações relacionadas à prevenção de doenças, promoção e recuperação da saúde, correlacionando seus saberes de enfermagem com os saberes da MTC. ${ }^{30}$

A adjetivação e especialização acupunturista enriquece o Ser Enfermeira, pois engloba conhecimentos da cultura oriental à expertise, e acrescentam ao cuidado experiências que focalizam os sentimentos e as emoções. Portanto, há conhecimento mais profundo e diversificado do cuidado, ampliando e solidificando a expertise, a autonomia sustentada pelo credencialismo descrito por Freidson.

No que se refere à integração, ainda há diferenças significativas nas concepções e escolhas dos diagnósticos de enfermagem, que envolvem o tipo de classificação, teorias e estratégias metodológicas. $O$ contexto da prática em evidências traz possibilidades teórico-metodológicas que contribuem para a prática da acupuntura. Observa-se pouca referência ao uso de uma classificação diagnóstica de enfermagem nos serviços de acupuntura. Os diagnósticos em acupuntura centram-se na estimulação de acupontos, atuando como regulador de fluxo da força vital (Qi) dentro do diagnóstico da Medicina Tradicional Chinesa, e liberação endógena e neuroquímica no tocante à clínica, que na enfermagem refletem os diagnósticos sobre as reações humanas advindas desses fluxos. Assim, os processos diagnósticos em acupuntura e em enfermagem são correspondentes. Nessa discussão, assume-se a acupuntura como uma prática que privilegia a intervenção de enfermagem. A organização do Processo de Enfermagem serve de auxílio à otimização da consulta em acupuntura. ${ }^{31}$

O diagnóstico de acupuntura é uma das tarefas mais intrincadas na Medicina Tradicional Chinesa (MTC). O processo de diagnóstico se baseia no princípio fundamental de que sinais e sintomas refletem as condições dos órgãos internos e dos canais. No processo de diagnóstico, a investigação deve estender-se para além de sinais e sintomas relacionados à queixa. Nesse sentido, histórico do paciente é uma etapa fundamental, pois busca sintomas subjetivos elucidados pelo paciente. ${ }^{25} \mathrm{~A}$ interface do Processo de Enfermagem e o diagnóstico em acupuntura amplia o conhecimento para a qualidade do cuidado à sociedade. Por ser uma tarefa de uso intensivo do conhecimento, de expertise, tem muito a contribuir para aprimorar ainda mais sua prática em acupuntura. ${ }^{13}$

Os cursos de graduação deveriam apontar para os estudantes as diversas áreas de atuação da enfermagem, sendo a acupuntura uma delas. Os estudantes precisam ter a oportunidade teórico-prática para correlacionar o exercício de enfermagem a áreas menos tradicionais, como as práticas 
alternativas. Vale a reflexão nas falas de alguns participantes, ao referirem que "aqui sou enfermeira" e "lá sou acupunturista". Ou seja, separam a profissão da especialização, quando na verdade uma complementa a outra na assistência praticada.

As limitações deste estudo mostram-se no âmbito metodológico, no sentido do alcance mais amplo de participantes, a impossibilidade de contato e indisponibilidade de alguns. Outra limitação referese aos poucos estudos nacionais e internacionais relacionados diretamente ao Processo de Enfermagem e diagnósticos e às práticas de registro em acupuntura. No entanto, o reconhecimento dessas limitações fortalece as narrativas e os resultados numa primeira análise.

\section{CONSIDERAÇÕES FINAIS}

O Processo de Enfermagem pode ser considerado um avanço aplicado na consulta de acupuntura, como também evolução no atendimento e estratégia de boa prática da enfermeira acupunturista. Frente ao exposto, e apesar de a enfermagem hoje se constituir uma das maiores e mais importantes profissões na área da saúde, no Brasil, e a acupuntura se apresentar como especialização dentro da enfermagem, ela vem ganhando destaque na profissão na área da saúde, em nível nacional.

Como potencialidade, destaca-se o avanço, para a área de enfermagem e na saúde, a utilização do Processo de Enfermagem no atendimento da enfermeira especialista em acupuntura fortalecendo a especialidade na área da enfermagem, de acordo com a sua regulamentação e inserção em políticas públicas de saúde, destacando a autonomia e a expertise de Freidson. Além disso, o estudo propicia o aumento da visibilidade do trabalho da enfermeira nessa especialidade.

A incorporação de tecnologias de cuidado na atenção à saúde mostra-se, para a enfermagem, potencial de qualificação da assistência, pois a acupuntura é evidenciada como tecnologia intervencionista, visando a resolutividade de necessidades humanas afetadas e reações que demonstrem desequilíbrio na condição de doença e de saúde.

Dessarte, sugerem-se outros estudos para aprofundar o motivo de que se reconhece a importância, mas não se utiliza o Processo de Enfermagem na prática da acupuntura, além de explorar as reais dificuldades em integrar os conhecimentos entre disciplinas correlatas.

\section{FINANCIAMENTO}

Ao CNPq, por possibilitar o desenvolvimento deste estudo. História da saúde e da enfermagem entre populações estigmatizadas e socialmente vulnerabilizadas, Processo: 304917/2019-2, Maria Itayra Coelho de Souza Padilha. O presente trabalho foi realizado com apoio da Coordenação de Aperfeiçoamento de Pessoal de Nível Superior - Brasil (CAPES) - Código de Financiamento 001, bolsa de mestrado a Ana Paula Senna Bousfield.

\section{CONTRIBUIÇÕES DOS AUTORES}

Desenho do estudo: Ana Paula Senna Bousfield. Maria Itayra Padilha.

Coleta ou produção dos dados: Ana Paula Senna Bousfield. Análise de dados: Ana Paula Senna Bousfield. Maria Itayra Padilha.

Interpretação dos resultados: Ana Paula Senna Bousfield. Maria Itayra Padilha.

Redação e revisão crítica do manuscrito. Ana Paula Senna Bousfield. Maria Itayra Padilha. Maria Lígia dos Reis Bellaguarda. Roberta Costa.

Aprovação da versão final do artigo. Ana Paula Senna Bousfield. Maria Itayra Padilha. Maria Lígia dos Reis Bellaguarda. Roberta Costa.

Responsabilidade por todos os aspectos do conteúdo e a integridade do artigo publicado. Ana Paula Senna Bousfield. Maria Itayra Padilha. Maria Lígia dos Reis Bellaguarda. Roberta Costa.

\section{EDITOR ASSOCIADO}

Marcelle Miranda da Silva

\section{REFERÊNCIAS}

1. Bulson JA, Bulson T. Nursing process and critical thinking Linked to disaster preparedness. J Emerg Nurs. 2011;37(5):477-83. http://dx.doi. org/10.1016/j.jen.2010.07.011. PMid:21889655.

2. Ribeiro OMPL, Martins MMFPS, Tronchin DMR, Forte ECN. Aplicação do processo de enfermagem em hospitais portugueses. Rev Gaúcha Enferm. 2018:39:e2017-0174. http://dx.doi.org/10.1590/1983-1447.2018.20170174.

3. Dell'Acqua MCQ, Miyadahira AMK. Ensino do processo de enfermagem nas escolas de graduação em enfermagem do estado de São Paulo. Rev. Latino-Am. Enfermagem. 2002 abr;10(2):185-91. http://dx.doi. org/10.1590/S0104-11692002000200010

4. Oliveira MR, Almeida PC, Moreira TMM, Torres RAM. Nursing care systematization: perceptions and knowledge of the Brazilian nursing. Rev Bras Enferm. 2019 dez;72(6):1547-53. http://dx.doi.org/10.1590/00347167-2018-0606. PMid:31644743.

5. Azevedo C, Moura CC, Corrêa HP, Mata LRF, Chaves ECL, Chianca TCM. Complementary and integrative therapies in the scope of nursing: legal aspects and academic-assistance panorama. Esc Anna Nery. 2019;23(2):e20180389. http://dx.doi.org/10.1590/2177-9465ean-2018-0389.

6. Resolução n. 0509/2016 (BR). Atualiza a norma técnica para Anotação de Responsabilidade Técnica pelo Serviço de Enfermagem e define as atribuições do enfermeiro Responsável Técnico. Diário Oficial da União, Brasília (DF), 2016.

7. de Sousa LA, de Barros NF. Integrative and Complementary Practices in the Unified Health System: progresses and challenges. Rev. LatinoAm. Enfermagem. 2018;26:e3041. http://dx.doi.org/10.1590/15188345.2854.3041.

8. Benedet S, Gelbcke F, Amante L, Padilha M, Pires D. Nursing process: systematization of the nursing care instrument in the perception of nurses. Revista de Pesquisa: Cuidado é Fundamental Online. 2016;8(3):47804788. http://dx.doi.org/10.9789/2175-5361.2016.v8i3.4780-4788.

9. Oliveira CS, Borges MS. Representações sociais de enfermeiros que cuidam de crianças sobre a sistematização da assistência de enfermagem. Rev Gaúcha Enferm. 2017;38(3):e66840. http://dx.doi. org/10.1590/1983-1447.2017.03.66840

10. Pereira RDM, Alvim NAT. Acupuntura para intervenção de diagnósticos de enfermagem: avaliação de experts e especialistas de enfermagem. 
Esc Anna Nery. 2016;20(4):e20160084. http://dx.doi.org/10.5935/14148145.20160084.

11. Cintra MER, Figueiredo R. Acupuntura e promoção de saúde: possibilidades no serviço público de saúde. Interface (Botucatu). 2010 mar;14(32):13954. http://dx.doi.org/10.1590/S1414-32832010000100012.

12. Rocha SP, Benedetto MACD, Fernandez FHB, Gallian DMC. A trajetória da introdução e regulamentação da acupuntura no Brasil: memórias de desafios e lutas. Ciênc. Saúde coletiva. 2015 Jan;20(1):155-64. http:// dx.doi.org/10.1590/1413-81232014201.18902013.

13. Bellaguarda MLR, Padilha MI, Nelson S. Eliot Freidson's sociology of professions: an interpretation for Health and Nursing. Rev Bras Enferm. 2020;73(6):e20180950. http://dx.doi.org/10.1590/0034-7167-2018-0950. PMid:32785504

14. Ministério da Saúde (BR). Secretaria de Atenção à Saúde. Política nacional de práticas integrativas e complementares no SUS: atitude de ampliação de acesso [Internet]. Brasília: Ministério da Saúde;2015 [citado 2019 jan 20]. Disponível em: http://bvsms.saude.gov.br/bvs/publicacoes/ politica_nacional_praticas_integrativas_complementares_2ed.pdf

15. Padilha MI, Bellaguarda MLR, Nelson S, Maia ARC, Costa R. O uso das fontes na condução da pesquisa histórica. Texto Contexto Enferm. 2017;26(4):e2760017. http://dx.doi.org/10.1590/0104-07072017002760017.

16. Meihy JCSB, Holanda F. História oral: como fazer, como pensar. São Paulo: Contexto; 2010

17. Minayo MCS. O desafio do conhecimento: pesquisa qualitativa em saúde. 14aㅡ ed. São Paulo: Hucitec; 2014.

18. Bitencourt JVOV, Martini JG, Massaroli A, Furlan de Léo MM, Conceição VM, Santos MG. Structuring of a proposal for the nursing precess theoretical and methdological basis: professor's motivations. Texto contexto - enferm. 2020 Dez;29:e20180205. http://dx.doi. org/10.1590/1980-265x-tce-2018-0205.

19. Lopes LF, Lopes MC, Fialho FAP, Gonçalves AL. Sistema de conhecimento para diagnóstico em acupuntura: uma modelagem usando o CommonKADS. Gestão \& Produção. 2011;18(2):351-66. http://dx.doi.org/10.1590/S0104-530X2011005000001.

20. Freidson E. Profissão médica: um estudo de sociologia do conhecimento aplicado. São Paulo: UNESP; 2009.

21. Bellaguarda MLR, Silveira LR, Mesquita MPL, Ramos FRS. Identidade da profissional enfermeira caracterizada numa revisão integrativa. Enf em Foco. 2011;2(3):180-3. http://dx.doi.org/10.21675/2357-707X.2011 v2.n3.130.

22. Benedet SA, Padilha MI, Gelbke FL, Bellaguarda MLR. The mode professionalism in the implementation of the Nursing Process (1979-2004). Rev Bras Enferm. 2018;71(4):1907-14. http://dx.doi.org/10.1590/00347167-2017-0226. PMid:30156676.

23. Kurebayashi LFS, Gnatta JR, Borges TP, Silva MJP. Avaliação diagnóstica da Medicina Tradicional Chinesa dos sintomas de estresse tratados pela auriculoterapia: ensaio clínico. Rev. Eletr. Enf. 2014 jan/mar;16(1):68-76. http://dx.doi.org/10.5216/ree.v16i1.20167.

24. Freidson E. Renascimento do profissionalismo: teoria, profecia e política. São Paulo: Edusp; 1988.

25. Bellaguarda ML, Nelson S, Padilha MI, Caravaca-Morera JA. Prescriptive Authority and Nursing: a comparative analysis of Brazil and Canada. Rev Lat Am Enfermagem. 2015;23(6):1065-73. http://dx.doi.org/10.1590/01041169.0418.2650. PMid:26625997.

26. Bousfield APS, Padilha MI, Martini JG, Nicácio AV. Inclusion of nurses in acupuncture practice in Santa Catarina (1997-2015). Cogitare enferm 2019;24:e66766. http://dx.doi.org/10.5380/ce.v24i0.66766.

27. Bellaguarda MLR, Padilha MI, Pereira Neto AF, Pires D, Peres MAA. Reflexão sobre a legitimidade da autonomia da enfermagem no campo das profissões de saúde à luz das ideias de Eliot Freidson. Esc Anna Nery. 2013;17(2):369-74. http://dx.doi.org/10.1590/S141481452013000200023.

28. Bellaguarda MLR, Padilha MI, Peres MAA, Paim L. Enfermagem profissão: seu status, eis a questão. Revista Enfermagem UERJ. 2016 abr;24(2):e8591. http://dx.doi.org/10.12957/reuerj.2016.8591.

29. Dallegrave $D$, Boff $C$, Kreutz JA. Acupuntura e Atenção Primária à Saúde: análise sobre necessidades de usuários e articulação da rede. Rev Bras Med Fam Comunidade. 2011;6(21):249-56. http://dx.doi. org/10.5712/rbmfc6(21)291.

30. Martins ES, Costa ND, Holanda SM, Castro RCMB, Aquino PDS, Pinheiro AKB. Enfermagem e a prática avançada da acupuntura para alívio da lombalgia gestacional. Acta Paul Enferm. 2019 out;32(5):47784. http://dx.doi.org/10.1590/1982-0194201900067.

31. Pereira RDM, Alvim AT, Pereira CD, Gomes JSC. Acupuntura na hipertensão arterial sistêmica e suas contribuições sobre diagnósticos de enfermagem. Esc Anna Nery. 2017;21(1):e20170024. http://dx.doi. org/10.5935/1414-8145.20170024.

a Artigo extraído de dissertação de mestrado, Título: Historicidade da prática da acupuntura por enfermeiras em Santa Catarina no período de 1997 a 2015 , de autoria de Ana Paula Senna Bousfield, orientado por: Maria Itayra Padilha, defendida no ano de 2018, Programa Pós-Graduação em Enfermagem da Universidade Federal de Santa Catarina. 